\title{
Pairing of weakly correlated electrons in the platinum-based centrosymmetric superconductor $\mathrm{SrPt}_{3} \mathrm{P}$
}

\author{
T. Shiroka, ${ }^{1,2,{ }^{*}}$ M. Pikulski, ${ }^{1}$ N. D. Zhigadlo, ${ }^{1}$ B. Batlogg, ${ }^{1}$ J. Mesot,${ }^{1,2}$ and H.-R. Ott ${ }^{1,2}$ \\ ${ }^{1}$ Laboratorium für Festkörperphysik, ETH Zürich, CH-8093 Zürich, Switzerland \\ ${ }^{2}$ Paul Scherrer Institut, CH-5232 Villigen PSI, Switzerland \\ (Received 18 February 2015; revised manuscript received 3 June 2015; published 18 June 2015)
}

\begin{abstract}
We report a study of the normal- and superconducting-state electronic properties of the centrosymmetric compound $\mathrm{SrPt}_{3} \mathrm{P}$ via ${ }^{31} \mathrm{P}$ nuclear-magnetic-resonance (NMR) and magnetometry investigations. Essential features such as a sharp drop of the Knight shift at $T<T_{c}$ and an exponential decrease of the NMR spin-lattice relaxation ratio $1 /\left(T_{1} T\right)$ below $T_{c}$ are consistent with an $s$-wave electron pairing in $\mathrm{SrPt}_{3} \mathrm{P}$, although a direct confirmation in the form of a Hebel-Slichter-type peak is lacking. Normal-state NMR data at $T<50 \mathrm{~K}$ indicate conventional features of the conduction electrons, typical of simple metals such as lithium or silver. Our data are finally compared with available NMR results for the noncentrosymmetric superconductors $\mathrm{LaPt}_{3} \mathrm{Si}_{\text {and }} \mathrm{CePt}_{3} \mathrm{Si}$, which adopt similar crystal structures.
\end{abstract}

DOI: 10.1103/PhysRevB.91.245143

PACS number(s): 74.25.nj, 74.70.Dd, 74.25.Ha, 76.60.-k

\section{INTRODUCTION}

The continuing search for new superconductors has recently resulted in the identification of a new family of superconducting phosphide compounds with the chemical composition $A \mathrm{Pt}_{3} \mathrm{P}(A=\mathrm{Sr}, \mathrm{Ca}$, or La) [1]. These materials, whose superconductivity has been claimed to be driven by conventional electron-phonon interactions, adopt a distorted antiperovskite structure, resembling the structure of several noncentrosymmetric superconductors, such as $\mathrm{LaPt}_{3} \mathrm{Si}$ or $\mathrm{CePt}_{3} \mathrm{Si}$ [2]. Contrary to the latter, however, $\mathrm{SrPt}_{3} \mathrm{P}$ exhibits an inversion center due to the staggered arrangement of $\mathrm{Pt}$ octahedra. Noncentrosymmetric superconductors, characterized by antisymmetric spin-orbit couplings, are currently the subject of intense research [3-6]. Therefore, a comparative study of their centrosymmetric counterparts is of particular interest. Indeed, the Sr-based compound of the new family, with a critical temperature $T_{c}=8.4 \mathrm{~K}$, has already been investigated in some detail. The first study included measurements of the specific heat $C(T)$ and Hall resistivity $\rho_{\mathrm{H}}(T)$ [1]. The specific-heat data were interpreted as indicating a strong electron-phonon coupling with a relatively large ratio $2 \Delta_{0} / k_{\mathrm{B}} T_{c} \sim 5$, and a fully gapped excitation spectrum below $T_{c}$ with a zero-temperature gap value $\Delta_{0}=1.85 \mathrm{meV}$. The nonlinear magnetic field dependence of Hall resistivity was attributed to the presence of multiple Fermi-surface pockets. Less details are known on $\mathrm{LaPt}_{3} \mathrm{P}$, whose critical temperature $T_{c} \simeq 1.5 \mathrm{~K}$ [1] is distinctly lower than that of the $\mathrm{Sr}$ compound, the difference being most likely due to the different valencies of the cations.

There exist several theoretical interpretations of the $\mathrm{SrPt}_{3} \mathrm{P}$ data, which differ in their conclusions. In Ref. [7], the proximity to a dynamical charge-density wave instability is claimed to favor the occurrence of superconductivity in $\mathrm{SrPt}_{3} \mathrm{P}$. In another approach, based on a Migdal-Eliashberg-type analysis, it is argued that a conventional phonon-mediated superconductivity is observed [8]. Density-functional-theory calculations [9] suggest that the onset of superconductivity is

\footnotetext{
*Corresponding author: tshiroka@ phys.ethz.ch
}

due to a strong coupling between the $p d \pi$-hybridized bands with low-energy phonon modes confined in the $a b$ plane of the crystal lattice. The two-dimensional character of these modes seems essential to preserve the antipolar arrangement of the distorted $\mathrm{Pt}$ octahedra in $\mathrm{SrPt}_{3} \mathrm{P}$, thus enhancing both the electron-phonon coupling constant $\lambda_{\text {ep }}$ and, consequently, the critical temperature $T_{c}$.

The only experimental investigation at a microscopic level cited in the literature focused on muon-spin rotation $(\mu \mathrm{SR})$ measurements [10]. The established temperature dependence of the penetration depth in the form of $\lambda^{-2}(T)$ again indicated a single gap with a value of $\Delta_{0}=1.55 \mathrm{meV}$. However, an upward curvature of the upper critical field $H_{c 2}(T)$ just below $T_{c}$ was interpreted as reflecting two-band superconductivity with equal gaps but differing values of the coherence lengths.

In this work we report on the microscopic physical properties of $\mathrm{SrPt}_{3} \mathrm{P}$, investigated by means of nuclear magnetic resonance $[11,12]$. The measurements described below are based on ${ }^{31} \mathrm{P}$ nuclear magnetic resonance (NMR), and probe both static (linewidths and line shifts), as well as dynamic (spin-lattice relaxation) properties of the material. Overall, the data indicate that $\mathrm{SrPt}_{3} \mathrm{P}$ is a rather simple metal and that the pairing configuration is most likely of spherically symmetric $(s$-wave) character. A comparison with the noncentrosymmetric superconductors $\mathrm{LaPt}_{3} \mathrm{Si}$ and $\mathrm{CePt}_{3} \mathrm{Si}$ is intended to clarify the peculiarities of the latter. The lack of inversion symmetry of the crystal lattice may partly account for the much lower $T_{c}$ of $\mathrm{LaPt}_{3} \mathrm{Si}$, while the rare-earth cations seem responsible for the unusual electronic properties of $\mathrm{CePt}_{3} \mathrm{Si}[3,5]$.

\section{EXPERIMENTAL DETAILS}

Polycrystalline samples of $\mathrm{SrPt}_{3} \mathrm{P}$ were synthesized under conditions of high pressure and high temperature. High-purity (99.99\%) coarse powders of $\mathrm{Sr}, \mathrm{Pt}$, and $\mathrm{P}$ were mixed in the stoichiometric 1:3:1 ratio, then thoroughly ground, and finally enclosed in a boron-nitride container. To avoid a possible degradation of the material due to air exposure, all the preparatory steps were done in a glovebox under argon atmosphere. For the heat treatment, the BN crucible 


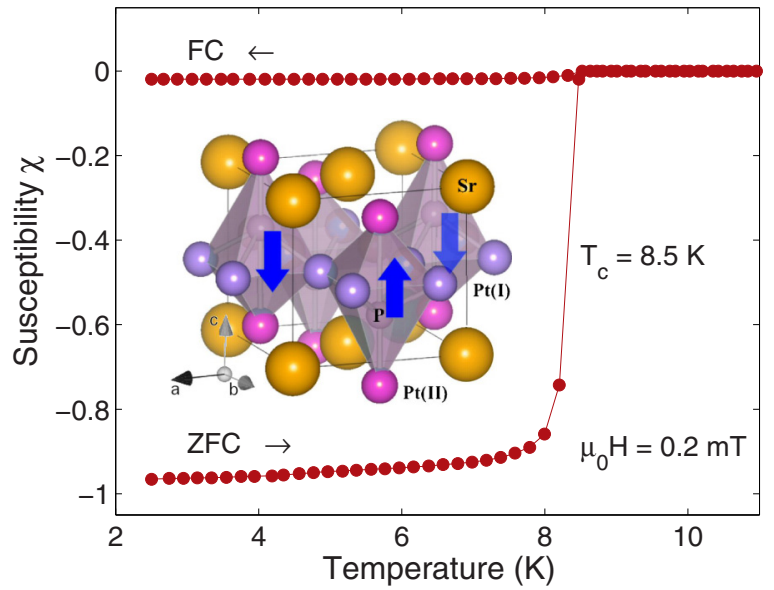

FIG. 1. (Color online) Temperature dependence of the zero-fieldcooled (ZFC) and field-cooled (FC) dc magnetization measured at $\mu_{0} H=0.2 \mathrm{mT}$. The sample shows a sizable diamagnetic response with $T_{c}=8.5 \mathrm{~K}$. Inset: structure of $\mathrm{SrPt}_{3} \mathrm{P}$ showing the $\mathrm{Pt}_{6}$ octahedra (adapted from Ref. [9]). The asymmetric positions of the apical Pt(II) ions give rise to alternating electric polarization vectors (blue arrows), hence preserving the centrosymmetric nature of $\mathrm{SrPt}_{3} \mathrm{P}$.

was placed into a pyrophyllite cube containing a graphite heater. An external pressure of $2 \mathrm{GPa}$ was applied at room temperature and kept constant during the $2 \mathrm{~h}$ ramping up of the temperature to $1050{ }^{\circ} \mathrm{C}$. The final temperature was kept constant for $40 \mathrm{~h}$ and then lowered back to room temperature within approximately $1 \mathrm{~h}$. After releasing the pressure, the polycrystalline samples were extracted and kept in closed capsules filled with Ar gas during the corresponding experiments. Subsequently recorded x-ray powder diffraction patterns agreed with those reported in Ref. [1]. Measurements of the low-temperature magnetization revealed the onset of a diamagnetic response at $T_{c}=8.5 \mathrm{~K}$, again in good agreement with the originally reported value [1]. The relevant magnetic susceptibility data $\chi(T)$ are shown in Fig. 1 where, at low temperatures, we observe a significant diamagnetic response, typical for the onset of superconductivity [13].

Although all the constituent elements of $\mathrm{SrPt}_{3} \mathrm{P}$ feature NMR-active nuclei, the ${ }^{31} \mathrm{P}$ nucleus is clearly the best choice with respect to isotopic abundance (100\%) and gyromagnetic ratio $(\gamma / 2 \pi=17.2356 \mathrm{MHz} / \mathrm{T})$. In addition, the $I=\frac{1}{2}$ nuclear spin of ${ }^{31} \mathrm{P}$ helps avoiding complications due to quadrupole effects. In order to probe an adequate region of the superconducting part of the $H-T$ phase diagram above $1.6 \mathrm{~K}$ (i.e., accessible with a ${ }^{4} \mathrm{He}$ flow cryostat), the external field for the NMR studies was set to $\mu_{0} H_{\text {ext }}=2 \mathrm{~T}$, corresponding to a critical temperature of approximately $5 \mathrm{~K}$ (see, e.g., Fig. 1 in Ref. [10]). Analogous measurements probing the normal state in the same temperature regime were made in a field of $\mu_{0} H_{\text {ext }}=7 \mathrm{~T}$, which exceeds the zero-temperature upper critical field $H_{c 2}(0) \simeq 5.5 \mathrm{~T}$ [10], and for monitoring the superconducting transition in a 0.6 - $\mathrm{T}$ field across a restricted temperature range around the relevant $T_{c}$.

In order to achieve an acceptable signal-to-noise $(\mathrm{S} / \mathrm{N})$ ratio, the sample was powdered to a grain size of the order of $10 \mu \mathrm{m}$ and kept in the form of a loose powder, hence reducing the electrical contact between the grains. NMR measurements at the above-mentioned external magnetic fields were made at different temperatures between 1.6 and $300 \mathrm{~K}$ by employing a conventional phase-coherent spectrometer. The ${ }^{31} \mathrm{P}$ spectra were obtained by fast Fourier transformation of the spin-echo signals generated by $\pi / 2-\pi$ rf pulses with a $200-\mu$ s delay between pulses (the measured transverse relaxation time $T_{2}$ is $\sim 500 \mu \mathrm{s})$. Since the employed "hard" $\mathrm{rf}$ pulses $\left(t_{\pi / 2} \sim 2.2 \mu \mathrm{s}\right)$ excite nonselectively the entire resonance line, no difference between fixed- and swept-frequency line shapes was observed. The nuclear spin-lattice relaxation times $T_{1}$ were measured at the peak position of each NMR line by means of the inversionrecovery method, using spin-echo detection at variable delays. The magnetic field was calibrated via ${ }^{27} \mathrm{Al}$ NMR of elemental aluminum, whose gyromagnetic ratio and Knight shift are known to high precision. This data was subsequently used to calculate the ${ }^{31} \mathrm{P}$ NMR shifts.

\section{EXPERIMENTAL RESULTS AND ANALYSIS}

\section{A. NMR line shapes and shifts}

In Fig. 2 we show the evolution of the ${ }^{31} \mathrm{P}$ lines with varying temperature at $\mu_{0} H=2.0 \mathrm{~T}$, both above and below $T_{c}$. Similar results with respect to the linewidths and line shifts were obtained for $\mu_{0} H=7.0 \mathrm{~T}$ (see Fig. 8).

We focus first on the normal phase. As can be seen in Fig. 3, the linewidth, of the order of $7 \mathrm{kHz}$ at room temperature, increases gradually by approximately $50 \%$ down to $\sim 12 \mathrm{~K}$, and subsequently it stays constant between $12 \mathrm{~K}$ and $T_{c}$. As for the line shifts in the normal phase, a moderate increase upon lowering the temperature is observed. This increase is

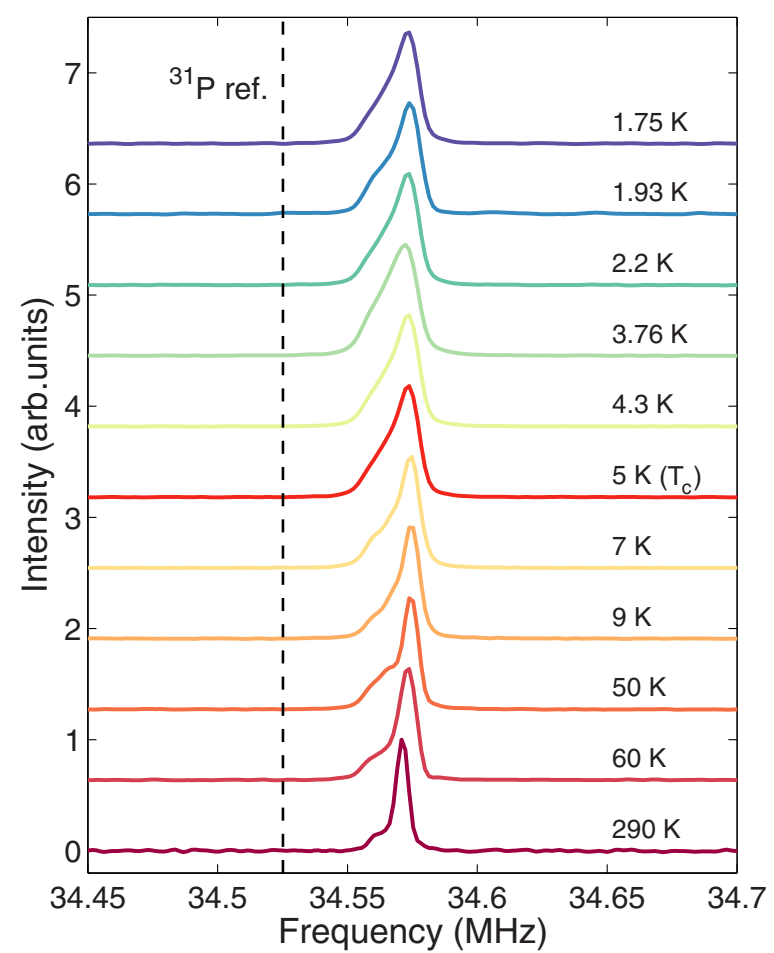

FIG. 2. (Color online) Representative ${ }^{31} \mathrm{P}$ NMR line shapes in $\mathrm{SrPt}_{3} \mathrm{P}$ at $\mu_{0} H=2.0 \mathrm{~T}$ and temperatures in the range $1.75-290 \mathrm{~K}$. The enhanced linewidths and asymmetry below $5 \mathrm{~K}$ reflect the onset of the superconducting phase. 


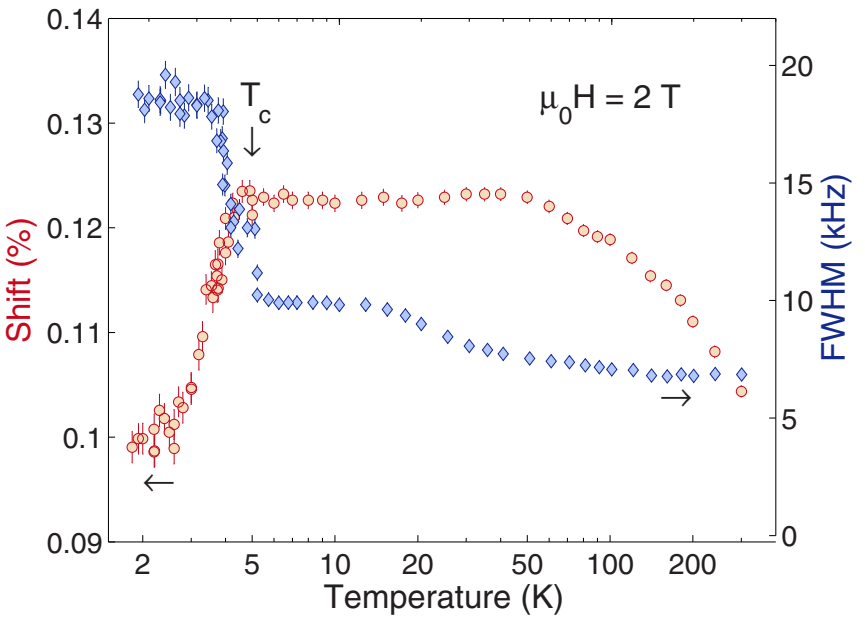

FIG. 3. (Color online) ${ }^{31} \mathrm{P}$ NMR shift (left scale) and linewidth (right scale) vs temperature, as measured at an applied field of $2 \mathrm{~T}$. The drop in shift and the increase in width below $T_{c}$ are followed by a constant behavior at intermediate temperatures and by a final decrease at higher $T$ (see text for details). The increase of data scattering below $T_{c}$ reflects the decreased $\mathrm{S} / \mathrm{N}$ ratio in the superconducting phase.

mostly linear down to approximately $50 \mathrm{~K}$, below which the line shift remains constant down to $T_{c}$ (see inset of Fig. 8). The constancy of the shift above the superconducting transition is a feature often observed in simple metals with an only weakly varying electronic susceptibility $\chi_{\mathrm{P}}(T)$. The relative shift, of the order of $0.12 \%$, is close to that observed in simple metals such as $\mathrm{Ag}$ and $\mathrm{Na}$ and is the same for the 2- and 7-T external fields, respectively (see Fig. 8). The linewidths measured in a field of $7 \mathrm{~T}$, instead, are approximately twice as large as those recorded at $\mu_{0} H=2 \mathrm{~T}$, which, in turn, are three times larger than those at $0.6 \mathrm{~T}$. Thus the linewidth scales almost linearly with the applied field.

The most interesting results are obtained below $T_{c}$, where the inhomogeneous field distribution in the mixed state of a type-II superconductor significantly broadens the NMR line. Figure 3 shows the steep increase of the linewidth, from 10 to $\sim 18 \mathrm{kHz}$, between $T_{c}$ and $3.5 \mathrm{~K}$ (and from 3.2 to $8 \mathrm{kHz}$ at 0.6 $\mathrm{T}-$ data not shown). Upon a further reduction of $T$, the width remains constant down to the lowest reached temperatures. At the same time the ${ }^{31} \mathrm{P}$ Knight shift exhibits a sudden decrease below $T_{c}$, a strong indication of spin-singlet superconductivity, since the pairing of electrons with opposite spins occurring in type $s$ - (or $d$-) wave superconductors implies a significant decrease of the local spin susceptibility, as detected by the probe nuclei. Of the two possibilities, the experimental data rule out the occurrence of a $d$-wave pairing, since in that case, the drop of the Knight shift would be less abrupt and the line broadening would reach its asymptotic $T=0 \mathrm{~K}$ value only gradually [11]. From these static NMR results, an $s$-type pairing seems to be the most plausible configuration adopted by electrons in the superconducting state of $\mathrm{SrPt}_{3} \mathrm{P}$.

\section{B. NMR relaxation rates and nature of superconductivity}

The conclusion about the $s$-wave pairing configuration is also supported by the ${ }^{31} \mathrm{P}$ spin-lattice $1 / T_{1}$ relaxation-rate data

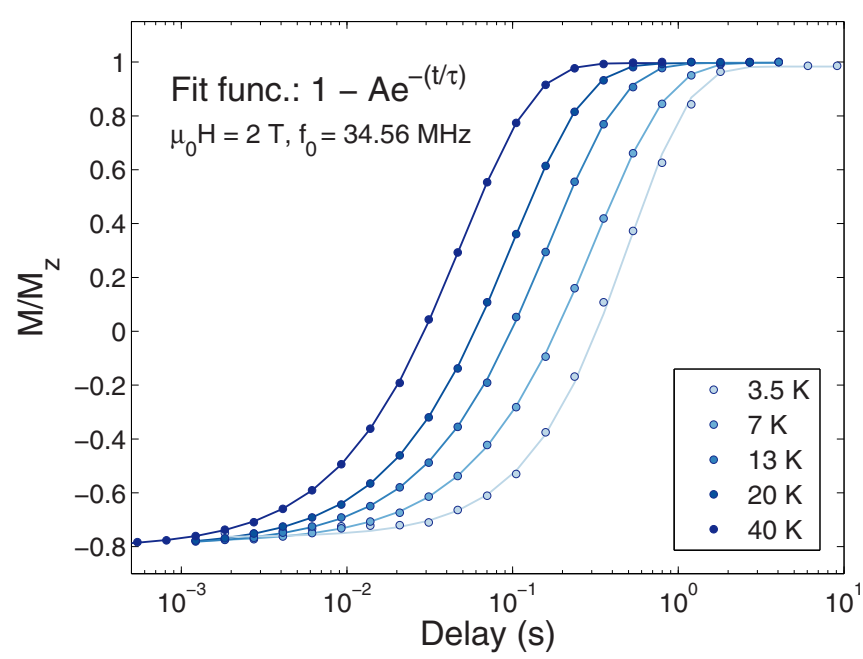

FIG. 4. (Color online) ${ }^{31} \mathrm{P}$ NMR spin-lattice relaxation data of $\mathrm{SrPt}_{3} \mathrm{P}$ at selected temperatures measured at $2 \mathrm{~T}$. All fit curves exhibit a simple exponential $(\beta=1)$ recovery of the magnetization (see text).

displayed in Fig. 5. The $1 /\left(T_{1} T\right)$ vs $T$ plot exhibits what is considered to be a typical signature of conventional BCS superconductivity, an exponential decrease with decreasing $T$ well within the superconducting phase, but no clear indication of a Hebel-Slichter-type coherence peak, which is masked by a distinct increase of $1 /\left(T_{1} T\right)$ in a narrow regime just above $T_{c}$.

The spin-lattice relaxation times $T_{1}$ were evaluated from magnetization-recovery curves such as those shown in Fig. 4. The nuclear magnetization data $M_{z}(t)$ were fitted by using the single-exponential equation valid for spin- $1 / 2$ nuclei:

$$
M_{z}(t)=M_{z}^{0}\left[1-f \exp \left(-t / T_{1}\right)^{\beta}\right] .
$$

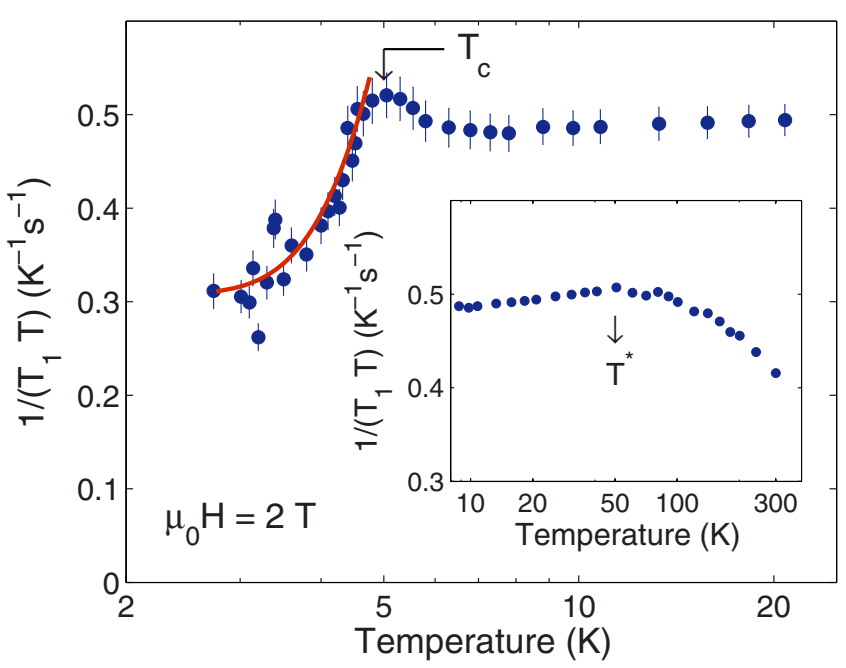

FIG. 5. (Color online) Temperature dependence of $\left(T_{1} T\right)^{-1}$ measured in a magnetic field of $2 \mathrm{~T}$. A similar cusplike feature at $T_{c}$ is seen also at $0.6 \mathrm{~T}$. The decrease below the maximum occurring at $T_{c}$ is compatible with a fully gapped superconductor (see text). Inset: $\left(T_{1} T\right)^{-1}$ data over the full temperature range show a broad maximum at $T^{*}=45 \mathrm{~K}$ (see also Fig. 7). 
Here $M_{z}^{0}$ represents the saturation value of magnetization at thermal equilibrium, $f$ is the inversion factor (exactly 2 for a complete inversion), and $\beta$ is a stretching exponent which accounts for possible distributions of relaxation rates [14]. Except at the lowest temperatures, the $\beta$ values were always found to be close to 1 , with no difference in fit quality whether $\beta$ was fixed or set free. Considering the purely magnetic relaxation of $I=1 / 2$ nuclear probes, this implies the same local environment for all the ${ }^{31} \mathrm{P}$ nuclei, i.e., disorder of either structural or magnetic nature is insignificant in our case.

Up to $T^{*}=45 \mathrm{~K}$, the normal-state spin-lattice relaxation rate varies approximately linearly with temperature (see inset in Fig. 5), as is usually observed in conventional simple metals. The progressive deviation from the linear-in- $T$ behavior above $T^{*}$ reflects a decrease in the dynamical spin susceptibility, as discussed in Secs. III C and III D.

Focusing back on the superconducting state, just below $T_{c}$ we do not observe a distinct Hebel-Slichter (coherence) peak, but only a cusplike feature peaking at $T_{c}$. Analogous data taken in a 0.6-T field showed essentially the same type of feature in the vicinity of $T_{c}$. We recall that the Hebel-Slichter anomaly is due to pair coherence and to a quasiparticle densityof-states anomaly at the gap edge and is more pronounced at lower fields, where the pair-breaking effect of the field is less important [15]. Both these effects can be weakened by different causes, including interaction anisotropies and the broadening of the quasiparticle states due to strong-coupling effects. Indeed, while weakly coupled conventional $s$-wave superconductors typically exhibit a Hebel-Slichter peak, this is not the case in the strong-coupling limit, where the enhanced electron-phonon coupling implies reduced quasiparticle lifetimes [16].

Since the lack of a coherence peak cannot rule out the possibility of an $s$-type electron pairing [17], further analysis is required. The temperature dependence of $T_{1}$ at $T<T_{c}$ can provide information about the symmetry of the superconducting gap. In the case of an anisotropic gap with nodes, a power-law dependence, $1 / T_{1} \sim T^{n}$, is expected since very low-energy electronic excitations around gap nodes can still contribute to relaxation. On the other hand, for standard fully gapped superconductors with no or a weak gap anisotropy, the relaxation rate depends exponentially on temperature, $1 / T_{1} \sim \exp \left(-\Delta_{0} / T\right)$, with $\Delta_{0}$ the superconducting gap at $T=0$. In either case, non-negligible $1 /\left(T_{1} T\right)$ residual values may be observed at the lowest temperatures [18]. Most likely these are due to the presence of vortex-core or of thermally excited vortex-motion relaxation mechanisms [19,20], which can be accounted for by an additional constant term. While both models provide reasonable fits to the data, the power-law expression shows a somehow poorer fit quality and, most importantly, the resulting exponent varies significantly (from 3.6 for the 2-T dataset to 2.7 for the data collected at $0.6 \mathrm{~T}$ ). On the other hand, as shown in Fig. 5, an exponential curve with $\Delta_{0}=1.47(4) \mathrm{meV}$ fits the $\mathrm{SrPt}_{3} \mathrm{P}$ relaxation data satisfactorily well. Also the data taken at $0.6 \mathrm{~T}$ can be consistently fitted with a similar gap value, $\Delta_{0}=1.51(4) \mathrm{meV}$, thus providing a good indication that the superconducting gap of $\mathrm{SrPt}_{3} \mathrm{P}$ is nodeless. The value of the gap parameter, although slightly modified by the presence of the constant term, is in fair agreement with the $1.55 \mathrm{meV}$ value reported in Ref. [10], but distinctly lower than

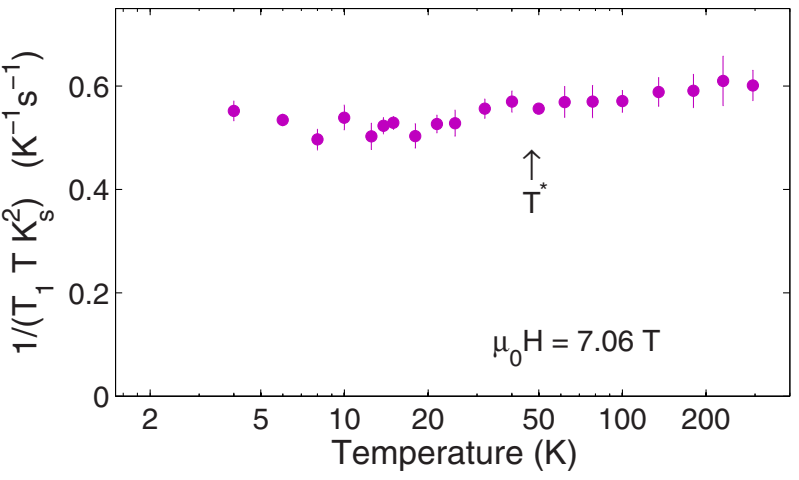

FIG. 6. (Color online) Korringa relation [reported in $S_{0}$ units; see Eq. (2)] shows only a weak temperature dependence, with no distinct features at $T^{*}=45 \mathrm{~K}$ (see also Fig. 7).

$\Delta_{0}=1.85 \mathrm{meV}$ reported in Ref. [1]. In any case, since the gap value from our analysis is clearly higher than $1.27 \mathrm{meV}$ (the gap expected from the BCS formula $2 \Delta_{0}=3.52 k_{\mathrm{B}} T_{c}$ ), it is compatible with a strong-coupling scenario.

Besides the vortex-core relaxation mechanism mentioned above, the almost constant value of $1 /\left(T_{1} T\right)$ well below $T_{c}$ might also reflect a certain degree of superconducting-gap anisotropy, but we consider this as a less likely possibility.

\section{Korringa relation and the degree of electronic correlation}

Some insight into the degree of electronic correlations in the normal state of a material can be gained by considering the so-called Korringa relation [21] (Fig. 6)

$$
T_{1} T K_{s}^{2}=S_{0}, \quad \text { with } \quad S_{0}=\frac{\gamma_{e}^{2}}{\gamma_{n}^{2}} \frac{\hbar}{4 \pi k_{\mathrm{B}}} .
$$

Here $\gamma_{e}$ and $\gamma_{n}$ are the electronic and the nuclear gyromagnetic ratios, respectively, while $K_{s}$ is the line shift due to the polarized conduction electrons. Equation (2) reflects the fact that both the readily accessible experimental parameters, the Knight shift $K_{s}$ and the spin-lattice relaxation time $T_{1}$, depend on the same electron-nucleus hyperfine interaction (assumed to be mostly of Fermi-contact character). However, while for a simple (Fermi-gas) metal the parameter $S_{0}$ is a constant (depending only on $\gamma_{n}$ of the probe nucleus), for real materials the generalized relation [22] $T_{1} T K_{s}^{2}=\alpha S_{0}$, with $\alpha$ a measure of the strength of the (Fermi-liquid) quasiparticle interactions [23], is more appropriate. Since $1 /\left(T_{1} T\right)$ probes the dynamical susceptibility averaged over the Brillouin zone, either ferromagnetic (FM) or antiferromagnetic spin correlations will enhance it, but only FM correlations can significantly enhance also the shift. Thus, $\alpha>1(<1)$ indicates the presence of ferro- (antiferro-) magnetic electronic correlations.

From the almost constant value of $S_{0} /\left(T_{1} T K_{s}^{2}\right)$ vs $T$ (see inset in Fig. 5) and considering the negligible orbital shift contribution (see below), we find $1 / \alpha \simeq 0.55$ and, hence, $\alpha=1.8$ in our case. The resulting value, slightly larger than unity, seems to suggest the presence of ferromagnetic correlations in the normal state. However, the ratio $1 / \alpha \simeq$ 0.55 is similar to $0.58,053$, and 0.50 observed in typical metals such as lithium, cesium, and silver, respectively [11]. 


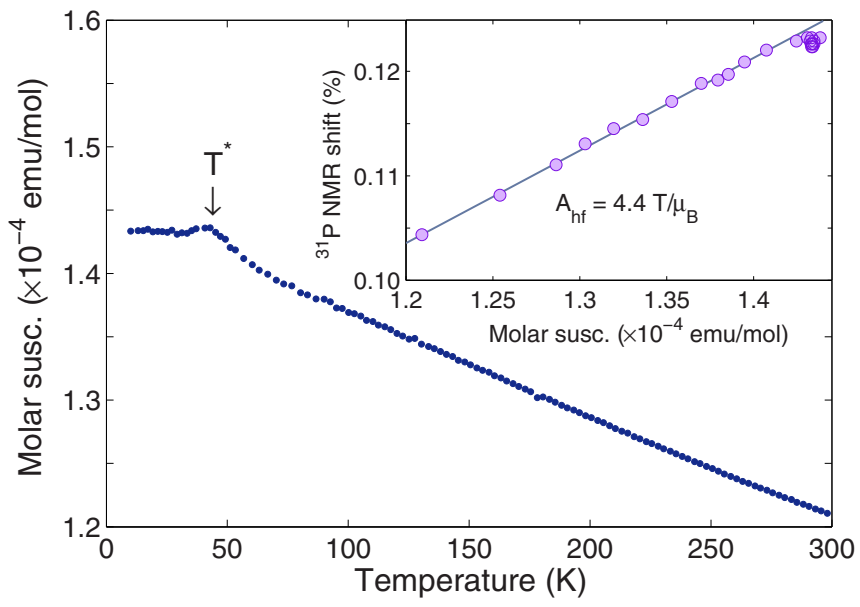

FIG. 7. (Color online) Temperature dependence of the $\mathrm{SrPt}_{3} \mathrm{P}$ molar magnetic susceptibility $\chi_{\text {mol }}$ in an applied field of $2 \mathrm{~T}$. Data were corrected by the addition of $1.18 \times 10^{-4} \mathrm{emu} / \mathrm{mol}$ to account for the core diamagnetism (see text). Inset: ${ }^{31} \mathrm{P}$ Clogston-Jaccarino $(K-\chi)$ plot at $\mu_{0} H=2 \mathrm{~T}$. The line is a fit to the $T>50 \mathrm{~K}$ data.

In these nearly-free-electron metals the Moriya theory of exchange enhancement, whereby the exchange fluctuations also enhance the rate of the $T_{1}$ process [11,24], explains the experimental data satisfactorily. Consequently, we conclude that also in $\mathrm{SrPt}_{3} \mathrm{P}$ the conduction electrons experience rather weak correlation effects, in good agreement with the results reported in Ref. [1].

\section{Magnetic susceptibility and NMR}

The magnetization $M(T)$ was measured in an applied magnetic field of $2 \mathrm{~T}$ in specimens from the same batch as that used for NMR experiments. The resulting values of the susceptibility, $\chi=M / H$, for temperatures above $10 \mathrm{~K}$ are shown in the main panel of Fig. 7. The measured net susceptibility seems unusually small for a metal, because the contribution from the conduction electrons is almost exactly canceled by the large core diamagnetism mainly from $\mathrm{Pt}$ $\left(1.18 \times 10^{-4} \mathrm{emu} / \mathrm{mol}\right.$ per formula unit, according to standard tables). This compensation effect makes it possible to measure even subtle variations of the electronic contribution with $T$.

In order to relate $\chi(T)$ with the NMR data, the relative ${ }^{31} \mathrm{P}$ NMR shifts in the two chosen magnetic fields (2 and $7 \mathrm{~T}$, respectively) are shown in Fig. 8. As expected for simple metals, the shifts are independent of the applied field and hence coincide perfectly. Below $T_{c}$ the high-field (7-T) data reflect the normal phase: both the line shift and linewidth remain practically constant upon decreasing temperature.

From the $\chi(T)$ data (shown in Fig. 7) and the NMR shifts (see inset of Fig. 8), one recognizes immediately very similar features. In either case, a practically linear increase upon lowering the temperature, from $300 \mathrm{~K}$ down to approximately $T^{*}=45 \mathrm{~K}$, is followed by a $T$-independent part. This clear break in the temperature dependence of both $K_{S}(T)$ and $\chi(T)$ coincides with a maximum of the Hall constant $R_{\mathrm{H}}$ reported in Ref. [1]. The reason for this might be that the trend to $R_{\mathrm{H}}=-1 /$ ne starts to dominate the $R_{\mathrm{H}}>0$ contribution.

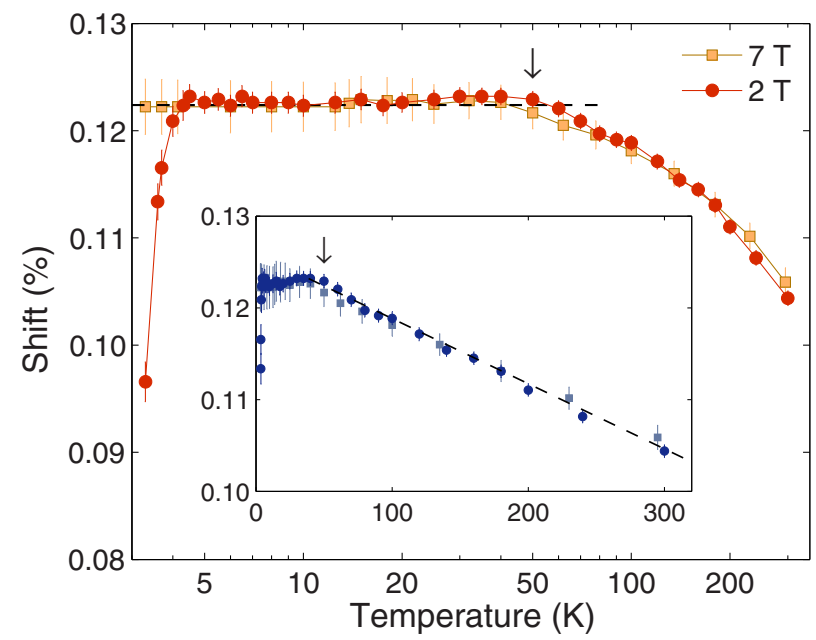

FIG. 8. (Color online) Comparison of ${ }^{31} \mathrm{P}$ NMR line shifts vs temperature at 2 (circles) and $7 \mathrm{~T}$ (squares) on logarithmic (main panel) and on linear scale (inset). The nature of the shift changes from constant to linearly decreasing at $45 \mathrm{~K}$ (arrows). The dashed lines highlight both regimes and serve as guides to the eye.

Such a comparison of microscopic (NMR) vs macroscopic (magnetometry) results provides further information about the normal-state electronic properties of $\mathrm{SrPt}_{3} \mathrm{P}$. In our case, given the relative insensitivity to the applied field (see Fig. 8), it makes sense to compare the NMR shifts with the susceptibility data, as shown in the Clogston-Jaccarino [25] (or $K-\chi$ ) plot in the inset of Fig. 7. Here both datasets refer to the same (2-T) field, with the temperature as an implicit parameter. From the slope $d K / d \chi$ of the $K-\chi$ plot for $T>50 \mathrm{~K}$, and by assuming a standard electronic $g$ factor of 2.0, the relation $K=g A_{\mathrm{hf}} \chi+K_{\text {orb }}$ gives the hyperfine coupling constant, $A_{\mathrm{hf}}=4.4 \mathrm{~T} / \mu_{\mathrm{B}}$, while the zero intercept with the $K$ axis gives the orbital shift, $K_{\text {orb }}=-0.003 \%$. The latter is perfectly compatible with known orbital-shift values for a light nucleus such as ${ }^{31} \mathrm{P}$. Clearly, the orbital shift is negligible in comparison with the dominant magnetic shift. We recall that hyperfine coupling constants are known to be strongly material dependent, so it is not unusual that our value is an order of magnitude higher than, e.g., $A_{\mathrm{hf}} \sim 0.2 \mathrm{~T} / \mu_{\mathrm{B}}$ found in another phosphorus-based compound, $\mathrm{Pb}_{2}(\mathrm{VO})\left(\mathrm{PO}_{4}\right)_{2}$ [26].

\section{DISCUSSION}

Below we discuss in more detail some aspects of the results presented in the previous sections.

Line shapes. For all the applied magnetic fields the normalstate ${ }^{31} \mathrm{P}$ lines show a slight asymmetry, usually attributed to the crystallite surfaces or to minor impurities. However, the persistence of the line asymmetry even in samples of different quality, most likely indicates a (uniaxial) anisotropy of the hyperfine coupling tensor (whose study is beyond the scope of this work), which can be observed also in powdered samples [27]. The uniaxial nature of the hf tensor is to be expected, if the symmetry of the distorted $\mathrm{Pt}$ octahedra probed by the phosphorus nuclei is considered (see structural unit in the inset of Fig. 1). 
The line shapes, in either the normal or the superconducting phase, indicate also the structural and chemical homogeneity of the sample. An essentially single line with a relatively narrow width of only $\sim 7 \mathrm{kHz}$ (see Fig. 2) is typical of defect-free crystal structures, especially when considering the random polycrystalline nature of the sample. Similarly, the line asymmetry observed below $T_{c}$, again reflects the high quality of the sample, since in a powdered superconductor with a significant degree of disorder the lines would appear symmetric.

Residual shift. A key prediction of the BCS theory for superconductors with singlet $s$-wave pairing is the vanishing of the Knight shift for $T \ll T_{c}$, reflecting the vanishing susceptibility due to the pairing of quasiparticles with antiparallel spins. Yet, even for elemental superconductors (as, e.g., Sn or $\mathrm{Hg}$ ), a residual shift is observed even close to $T=0$ [19]. The presence of a significant orbital Knight shift and/or of spin-orbit scattering (known as Ferrell-Anderson effect) have been identified as responsible for its occurrence. The $\mathrm{SrPt}_{3} \mathrm{P}$ case, however, is a bit more complex, since here ${ }^{31} \mathrm{P}$ is a low- $Z$ nucleus which interacts with superconducting carriers arising mostly from in-plane $p d \pi$-hybridized states between Pt and P ions [9]. Since orbital shifts are negligible for the light probe nuclei, the presence of a low-temperature nonzero Knight shift in $\mathrm{SrPt}_{3} \mathrm{P}$ is, therefore, most likely due to spin-orbit scattering effects.

Core-polarization effects and $T^{*}$ anomaly. Another contribution to the nonvanishing shift at $T=0$ can arise from corepolarization effects $[19,28]$, whereby the exchange interaction of localized core electrons with field-polarized conduction band electrons (partially of $d$ character in our case), implies an additional nonzero contact interaction in the ${ }^{31} \mathrm{P}$ nuclei. The observation of large negative ${ }^{195} \mathrm{Pt}-\mathrm{NMR}$ Knight shifts (data not shown) seems to confirm the presence of core-polarization effects in $\mathrm{SrPt}_{3} \mathrm{P}$ [25]. While this contribution to the Knight shift would disappear entirely if purely $d$-band electrons were coupled into Cooper pairs, a residual shift would still arise considering the hybridized (i.e., not exclusively $d$ ) character of the conduction band quasiparticles.

The consequences of core-polarization effects are more important, however, in the normal state. The electronic contribution to the magnetic susceptibility of a $d$-type band is generally temperature dependent, thus implying a temperaturedependent Knight shift, as actually observed (for an ideal metal one would have expected a constant shift). While corepolarization effects might explain the similar $T$ dependence of $K$ and $\chi_{s}$, the abrupt change in slope observed at $T^{*}$, as well as the value of $T^{*}$ itself, remain to be investigated.

Weak electron correlation. Our NMR data of $\operatorname{SrPt}_{3} \mathrm{P}$, indicate a fairly simple metallic behavior in the normal state, followed by standard BCS features in the superconducting state. These results are in remarkable agreement with the transport and magnetometry measurements reported in Ref. [1] in two important aspects. First, both NMR (via the Korringa product) and the macroscopic measurements (via the Sommerfeld-Wilson ratio [29]) demonstrate the absence of significant electron correlations. Secondly, there is a distinct feature at $T^{*}=45 \mathrm{~K}$ in the ${ }^{31} \mathrm{P}$ Knight shift and in the $1 /\left(T_{1} T\right)$ relaxation behavior, respectively, both of which change from a constant-in- $T$ to a linear-in- $T$ decrease as the temperature changes across $T^{*}$. This temperature coincides with the above-mentioned maximum in $R_{\mathrm{H}}(T)$, the latter reflecting anisotropies of the electron mean free path across different parts of the Fermi surface. The latter fact is consistent with conclusions drawn in Ref. [10]. The coincidence of $T^{*}$ values, derived from results of very different types of experiments, is unclear at present.

As for the Korringa relation, we recall that the absolute value of $\alpha$ might also be influenced by extraneous factors, such as the presence of strong disorder [30], or effects related to the hyperfine form factor (i.e., $q$-space filtering). Nevertheless, as mentioned above, these can be excluded in our case and the observation of a constant Korringa ratio vs temperature represents a valid proof of Fermi-liquid behavior in $\mathrm{SrPt}_{3} \mathrm{P}$, where $1 / \alpha \simeq 0.55$ is constant over a fairly wide temperature range.

Centro- vs noncentro-symmetric superconductors. We conclude with a comparison of $\mathrm{SrPt}_{3} \mathrm{P}$ with two noncentrosymmetric superconductors, the nonmagnetic $\mathrm{LaPt}_{3} \mathrm{Si}\left(T_{c}=0.6 \mathrm{~K}\right)$ and the magnetic $\mathrm{CePt}_{3} \mathrm{Si}\left(T_{c}=0.75 \mathrm{~K}\right)$, both of which were studied via NMR, as well [3-5]. The latter not only share the same structure, but show also similar antisymmetric spin-orbit couplings. Hence, a comparison of the three is relevant to understand whether it is the lack of inversion symmetry, or the presence of rare-earth ions to account for the unusual properties of noncentrosymmetric superconductors.

While $\mathrm{SrPt}_{3} \mathrm{P}$ and $\mathrm{LaPt}_{3} \mathrm{Si}$ both follow a perfect Korringa law down to $T_{c}$, in $\mathrm{CePt}_{3} \mathrm{Si}$ the $1 /\left(T_{1} T\right)$ ratio is enhanced upon cooling due to the development of $4 f$-derived magnetic fluctuations. Most importantly, unlike in $\mathrm{SrPt}_{3} \mathrm{P}$ and $\mathrm{LaPt}_{3} \mathrm{Si}$, where the superconducting transition is of conventional type and not dominated by correlation effects, in $\mathrm{CePt}_{3} \mathrm{Si}$, the superconductivity emerges from a unique heavy-electron state which coexists with antiferromagnetic order.

From these facts, we conclude that the key differences between the considered centrosymmetric and noncentrosymmetric superconductors, are most likely related to the presence of $\mathrm{Ce}^{3+}$ ions and the ensuing heavy-fermion phenomena, while the parity mixing alone (common to both $\mathrm{LaPt}_{3} \mathrm{Si}$ and $\mathrm{CePt}_{3} \mathrm{Si}$ ) cannot explain the peculiar behavior of the latter. Despite the many similarities between $\mathrm{SrPt}_{3} \mathrm{P}$ and $\mathrm{LaPt}_{3} \mathrm{Si}$, their belonging to different classes of crystal symmetry may possibly explain their distinctly different critical temperatures. Incidentally, from the available data in literature, also the centrosymmetric $\mathrm{LaPt}_{3} \mathrm{P}$ compound seems most likely to be a conventional BCS type-I superconductor.

\section{CONCLUSIONS}

We presented the results of ${ }^{31} \mathrm{P}$ NMR measurements in the recently discovered $\mathrm{SrPt}_{3} \mathrm{P}$ superconducting compound. In the normal state, the nuclear spin-relaxation rate obeys a Korringa-type relation $\left(T_{1} T\right)_{n}^{-1}=0.522(\mathrm{sK})^{-1}$ and the line shift is independent of temperature. In the superconducting state, the lack of a clear coherence peak in $T_{1}^{-1}$ just below $T_{c}$, most likely due to a strong electron-phonon coupling, is followed by an exponential, thermally activated decrease upon further cooling. A similar behavior is shown also by the line shift. The overall results of our NMR investigation make us conclude that $\mathrm{SrPt}_{3} \mathrm{P}$, which behaves as a standard metal in the normal state, is characterized by singlet pairing of the electrons 
and a gap function with conventional $s$-wave-type symmetry in the superconducting state.

Finally, a comparison with the analogous noncentrosymmetric superconductors $\mathrm{LaPt}_{3} \mathrm{Si}$ and $\mathrm{CePt}_{3} \mathrm{Si}$, strongly suggests that the peculiarities of the latter are closely related to the presence of rare-earth cations. The lack of inversion symmetry may cause a much lower $T_{c}$, however.

\section{ACKNOWLEDGMENTS}

The authors thank R. Khasanov for sharing his data prior to publication and for useful discussions. This work was financially supported in part by the Schweizerische Nationalfonds zur Förderung der Wissenschaftlichen Forschung (SNF) and the NCCR research pool MaNEP of SNF.
[1] T. Takayama, K. Kuwano, D. Hirai, Y. Katsura, A. Yamamoto, and $\mathrm{H}$. Takagi, Strong coupling superconductivity at $8.4 \mathrm{~K}$ in an antiperovskite phosphide $\mathrm{SrPt}_{3} \mathrm{P}$, Phys. Rev. Lett. 108, 237001 (2012).

[2] E. Bauer, R. Lackner, G. Hilscher, H. Michor, M. Sieberer, A. Eichler, A. Gribanov, Y. Seropegin, and P. Rogl, REPt ${ }_{3} \mathrm{Si}$ $(\mathrm{RE}=\mathrm{La}, \mathrm{Pr}, \mathrm{Nd}, \mathrm{Sm}$ and $\mathrm{Gd})$ : Isotypes of the heavy fermion superconductor $\mathrm{CePt}_{3} \mathrm{Si}$, J. Phys.: Condens. Matter 17, 1877 (2005).

[3] M. Yogi, Y. Kitaoka, S. Hashimoto, T. Yasuda, R. Settai, T. D. Matsuda, Y. Haga, Y. Ōnuki, P. Rogl, and E. Bauer, Evidence for a novel state of superconductivity in noncentrosymmetric $\mathrm{CePt}_{3} \mathrm{Si}$ : A ${ }^{195} \mathrm{Pt}-\mathrm{NMR}$ study, Phys. Rev. Lett. 93, 027003 (2004).

[4] M. Yogi, Y. Kitaoka, S. Hashimoto, T. Yasuda, R. Settai, T. D. Matsuda, Y. Haga, Y. Ōnuki, P. Rogl, and E. Bauer, ${ }^{195} \mathrm{Pt}-\mathrm{NMR}$ study on noncentrosymmetric heavy-fermion superconductor $\mathrm{CePt}_{3} \mathrm{Si}$, J. Phys. Chem. Solids 67, 522 (2006).

[5] H. Mukuda, S. Nishide, A. Harada, K. Iwasaki, M. Yogi, M. Yashima, Y. Kitaoka, M. Tsujino, T. Takeuchi, R. Settai, Y. Ōnuki, E. Bauer, K. M. Itoh, and E. E. Haller, Multiband superconductivity in heavy-fermion compound $\mathrm{CePt}_{3} \mathrm{Si}$ without inversion symmetry: An NMR study on a high-quality single crystal, J. Phys. Soc. Jpn. 78, 014705 (2009).

[6] Non-Centrosymmetric Superconductors, edited by E. Bauer and M. Sigrist, Lecture Notes in Physics Vol. 847 (Springer, Berlin, 2012).

[7] H. Chen, X.-F. Xu, C. Cao, and J. Dai, First-principles calculations of the electronic and phonon properties of $A \mathrm{Pt}_{3} \mathrm{P}(A=\mathrm{Ca}$, $\mathrm{Sr}$, and $\mathrm{La}$ ): Evidence for a charge-density-wave instability and a soft phonon, Phys. Rev. B 86, 125116 (2012).

[8] A. Subedi, L. Ortenzi, and L. Boeri, Electron-phonon superconductivity in $A \mathrm{Pt}_{3} \mathrm{P}$ ( $\left.A=\mathrm{Sr}, \mathrm{Ca}, \mathrm{La}\right)$ compounds: From weak to strong coupling, Phys. Rev. B 87, 144504 (2013).

[9] C.-J. Kang, K.-H. Ahn, K.-W. Lee, and B. I. Min, Electron and phonon band-structure calculations for the antipolar $\mathrm{SrPt}_{3} \mathrm{P}$ antiperovskite superconductor: Evidence of low-energy twodimensional phonons, J. Phys. Soc. Jpn. 82, 053703 (2013).

[10] R. Khasanov, A. Amato, P. K. Biswas, H. Luetkens, N. D. Zhigadlo, and B. Batlogg, $\mathrm{SrPt}_{3} \mathrm{P}$ : A two-band single-gap superconductor, Phys. Rev. B 90, 140507(R) (2014).

[11] R. E. Walstedt, The NMR Probe of High-T Materials (Springer Verlag, Berlin, 2008).

[12] N. J. Curro, Nuclear magnetic resonance in the heavy fermion superconductors, Rep. Prog. Phys. 72, 026502 (2009).

[13] Since the magnetic response of a superconductor is influenced by many factors, such as the sample granularity, its compactness, flux pinning, the demagnetization factor, the remnant field, etc., the proximity to the ideal diamagnetic response is rather coincidental in our case.
[14] T. Shiroka, F. Casola, V. Glazkov, A. Zheludev, K. Prša, H.-R. Ott, and J. Mesot, Distribution of NMR Relaxations in a Random Heisenberg Chain, Phys. Rev. Lett. 106, 137202 (2011).

[15] Y. Masuda and N. Okubo, Nuclear spin-lattice relaxation in superconducting mixed state, J. Phys. Soc. Jpn. 26, 309 (1969).

[16] Sh. Ohsugi, Y. Kitaoka, M. Kyogaku, K. Ishida, K. Asayama, and T. Ohtani, Nuclear relaxation study in strong coupling superconductors-A comparison with high- $T_{c}$ superconductors, J. Phys. Soc. Jpn. 61, 3054 (1992).

[17] D. Parker, O. V. Dolgov, M. M. Korshunov, A. A. Golubov, and I. I. Mazin, Extended $s_{ \pm}$scenario for the nuclear spin-lattice relaxation rate in superconducting pnictides, Phys. Rev. B 78, 134524 (2008).

[18] Since samples of different quality show similar residual values, the observed offset in $1 /\left(T_{1} T\right)$ is likely of intrinsic origin.

[19] D. E. MacLaughlin, Magnetic resonance in the superconducting state, in Solid State Physics, edited by H. Ehrenreich, F. Seitz, and D. Turnbull (Academic, New York, 1976), Vol. 31, pp. 1-69.

[20] M. Corti, B. J. Suh, F. Tabak, A. Rigamonti, F. Borsa, M. Xu, and B. Dabrowski, Flux-line dynamics in $\mathrm{YBa}_{2} \mathrm{Cu}_{4} \mathrm{O}_{8}$ from ${ }^{89} \mathrm{Y}$ NMR, Phys. Rev. B 54, 9469 (1996).

[21] J. Korringa, Nuclear magnetic relaxation and resonance line shift in metals, Physica 16, 601 (1950).

[22] A. Narath and H. T. Weaver, Effects of electron-electron interactions on nuclear spin-lattice relaxation rates and Knight shifts in alkali and noble metals, Phys. Rev. 175, 373 (1968).

[23] D. Pines, Paramagnetic susceptibility of conduction electrons, Phys. Rev. 95, 1090 (1954).

[24] T. Moriya, The effect of electron-electron interaction on the nuclear spin relaxation in metals, J. Phys. Soc. Jpn. 18, 516 (1963).

[25] A. M. Clogston, V. Jaccarino, and Y. Yafet, Interpretation of Knight shifts and susceptibilities of transition metals: Platinum, Phys. Rev. 134, A650 (1964).

[26] R. Nath, Y. Furukawa, F. Borsa, E. E. Kaul, M. Baenitz, C. Geibel, and D. C. Johnston, Single-crystal ${ }^{31} \mathrm{P}$ NMR studies of the frustrated square-lattice compound $\mathrm{Pb}_{2} \mathrm{VO}\left(\mathrm{PO}_{4}\right)_{2}$, Phys. Rev. B 80, 214430 (2009).

[27] A. Kawamoto, K. Miyagawa, Y. Nakazawa, and K. Kanoda, ${ }^{13} \mathrm{C}$ NMR study of layered organic superconductors based on BEDT-TTF molecules, Phys. Rev. Lett. 74, 3455 (1995).

[28] A. J. Freeman and R. E. Watson, Hyperfine interactions in magnetic materials, in Magnetism, edited by G. T. Rado and H. Suhl (Academic, New York, 1965), Vol. 2A, pp. 168-305.

[29] The Sommerfeld-Wilson ratio, the dimensionless ratio of the zero-temperature magnetic susceptibility, and the linearly varying term of the temperature dependence of specific heat, can provide insight into the degree of electronic correlation.

[30] B. S. Shastry and E. Abrahams, What Does the Korringa Ratio Measure?, Phys. Rev. Lett. 72, 1933 (1994). 